\title{
EXISTENCE AND UNIQUENESS OF SOLUTIONS FOR TWO- DIMENSIONAL FRACTIONAL NON- COLLIDING PARTICLE SYSTEMS
}

\author{
Vu Thi Huong ${ }^{1}$ \\ ${ }^{1}$ University of Transport and Communications, No 3 Cau Giay Street, Hanoi, Vietnam.
}

\section{ARTICLE INFO}

TYPE: Research Article

Received: 5/11/2019

Revised: 2/12/2019

Accepted: 5/12/2019

Published online: $31 / 1 / 2020$

https://doi.org/10.25073/tcsj.71.1.2

${ }^{*}$ Corresponding author

Email: vthuong@utc.edu.vn; Tel: 0902832246

Abstract. In this paper, we consider the stochastic evolution of two particles with electrostatic repulsion and restoring force which is modeled by a system of stochastic differential equations driven by fractional Brownian motion where the diffusion coefficients are constant. This is the simplest case for some classes of non- colliding particle systems such as Dyson Brownian motions, Brownian particles systems with nearest neighbour repulsion. We will prove that the equation has a unique non- colliding solution in path- wise sense.

Keywords: stochastic differential equation, fractional Brownian motion, non- colliding particle systems.

(C) 2020 University of Transport and Communications

\section{INTRODUCTION}

It is known that the systems of SDEs driven by standard Brownian motion describing positions of $d$ ordered particles evolving in $\mathrm{R}$ has the form

$$
d x_{i}(t)=\left\{\sum_{j \neq i} \frac{\gamma_{i j}}{x_{i}(t)-x_{j}(t)}+b_{i}(t, x(t))\right\} d t+\sum_{j=1}^{m} \sigma_{i j}(x(t)) d W_{j}(t), i=1, \ldots d,
$$

where $W=\left(W_{1}(t), W_{2}(t), \ldots, W_{m}(t)\right)$ is a $m$ - dimensional standard Brownian. The system of SDEs (2) is a type of SDEs whose solution stays in a domain which has been studied by many 
authors because of its important applications in physics, biology and finance [1]. In mathematical physics, the process $x(t)$ is used to model systems of $d$ non-colliding particles with electrostatic repulsion and restoring force. It contains Dyson Brownian Motions, Squared Bessel particle systems, Jacobi particle systems, non-colliding Brownian and Squared Bessel particles, potential-interacting Brownian particles and other particle systems crucial in mathematical physics and physical statistics $[2,3]$. The existence and uniqueness of a strong non-colliding solution to such kind of systems have been intensively studied by many authors $([4,5,6,7]$ and the references therein). But there are no results in the case of fractional noncolliding particles.

The main aim of this paper is to study the two- dimensional fractional noncolliding particle systems

$$
\left\{\begin{array}{l}
d X_{1}(t)=\left(\frac{\gamma}{X_{1}(t)-X_{2}(t)}+b_{1}(t, X(t))\right) d t+\sum_{j=1}^{m} \sigma_{1 j} d B_{j}^{H}(t), \\
d X_{2}(t)=\left(\frac{\gamma}{X_{2}(t)-X_{1}(t)}+b_{2}(t, X(t))\right) d t+\sum_{j=1}^{m} \sigma_{2 j} d B_{j}^{H}(t) .
\end{array}\right.
$$

where $X(0)=\left(X_{1}(0), X_{2}(0)\right) \in \Delta_{2}=\left\{x=\left(x_{1}, x_{2}\right)^{T} \in \mathbb{R}^{2}: x_{1}<x_{2}\right\}$ almost surely (a.s) and $B=\left\{B^{H}(t), t \geq 0\right\}=\left(B_{1}^{H}(t), B_{2}^{H}(t), \ldots, B_{m}^{H}(t)\right)^{T}$ is an $m$-dimensional fractional Brownian motion with the Hurst parameter $H \in\left(\frac{1}{2}, 1\right)$ defined on a complete probability space $(\Omega, \mathcal{F}, P)$ with a filtration $\left\{\mathcal{F}_{t}, t \geq 0\right\}$ satisfying the usual conditions. We prove that equation (1) has a unique non- colliding solution in path-wise sense. To the best of my knowledge, this is the first paper to discuss the fractional non- colliding particle systems.

\section{THE EXISTENCE AND UNIQUENESS OF THE SOLUTION}

Fix $T>0$ and we consider eq. (1) on the interval $[0, T]$. We suppose that the coefficients $b_{i}:[0 ;+\infty) \times \mathbb{R}^{2} \rightarrow \mathbb{R}$ are measurable functions and there exist positive constants $L, C$ such that following conditions hold

(i) $X(0) \in \Delta_{2}$ almost surely.

(ii) $\gamma>0$.

(iii) $b_{i}(\mathrm{t}, \mathrm{x}), i=1,2$ are globally Lipschitz continuous with respect to $x$, that is

$$
\sup _{i=1,2}\left|b_{i}(t, x)-b_{i}(t, y)\right| \leq L|x-y|,
$$

for all $x, y \in \mathbb{R}^{2}$ and $t \in[0, T]$.

(iv) $b_{i}(t, x), i=1,2$ are sub-linearly growth with respect to $x$, that is

$$
\sup _{i=1,2}\left|b_{i}(t, x)\right| \leq C(1+|x|) \text {, }
$$

for all $x \in \mathbb{R}^{2}$ and $t \in[0, T]$.

(v) $b_{1}(t, x)<b_{2}(t, x)$ for all $x \in \mathbb{R}^{2}$ and $t \in[0, T]$. 
Transport and Communications Science Journal, Vol. 71, Issue 1 (01/2020), 11-17

Denote $a \vee b=\max \{a, b\}$ and $a \wedge b=\min \{a, b\}$. For each $n \in \mathbb{N}$, we consider the following fractional SDEs

$$
\left\{\begin{array}{l}
d X_{1}^{n}(t)=\left(\frac{\gamma}{\left(X_{1}^{n}(t)-X_{2}^{n}(t)\right) \wedge \frac{-1}{n}}+b_{1}\left(t, X^{n}(t)\right) d t+\sum_{j=1}^{m} \sigma_{1 j} d B_{j}^{H}(t),\right. \\
d X_{2}^{n}(t)=\left(\frac{\gamma}{\left(X_{2}^{n}(t)-X_{1}^{n}(t)\right) \vee \frac{1}{n}}+b_{2}\left(t, X^{n}(t)\right)\right) d t+\sum_{j=1}^{m} \sigma_{2 j} d B_{j}^{H}(t),
\end{array}\right.
$$

where $X^{n}(0)=\left(X_{1}^{n}(0), X_{2}^{n}(0)\right) \in \Delta_{2}$. For each $n \in \mathbb{N}$ and $x=\left(x_{1}, x_{2}\right)$ we set

$$
\begin{aligned}
& f_{1}^{n}(t, x)=\frac{\gamma}{\left(x_{1}-x_{2}\right) \wedge \frac{-1}{n}}+b_{1}(t, x), \\
& f_{2}^{n}(t, x)=\frac{\gamma}{\left(x_{2}-x_{1}\right) \vee \frac{1}{n}}+b_{2}(t, x) .
\end{aligned}
$$

Lemma 2.1. For each $T>0$, eq. (3) has a unique solution on [0,T].

Proof: Using the estimate $|a \vee c-b \vee c| \leq|a-b|,|a \wedge c-b \wedge c| \leq|a-b|$, it is straightforward to verify that

$$
\left|f_{i}^{n}(t, x)-f_{i}^{n}(t, y)\right| \leq\left(\sqrt{2} \gamma n^{2}+L\right)|x-y|
$$

for all $x=\left(x_{1}, x_{2}\right)$ and $t \in[0, T]$ and

$$
\left|f_{i}^{n}(t, x)\right| \leq n \gamma+C(1+|x|) \text {. }
$$

It means that coefficients of eq. (3) satisfy Lipschitz continuity and boundedness condition. Hence it follows from Theorem 2.1 in [8] that eq. (3) has a unique solution on the interval $[0, T]$.

We recall a result on the modulus of continuity of trajectories of fractional Brownian motion ([9])

Lemma 2.2. Let $B=\left\{B^{H}(t), t \geq 0\right\}$ be a fractional Brownian motion of Hurst parameter $H \in(0,1)$. Then for every $0<\varepsilon<H$ and $T>0$, there exists an event $\Omega_{\varepsilon, T}$ with $P\left(\Omega_{\varepsilon, T}\right)=1$, and a positive random variable $\eta_{\varepsilon, T}$ such that $\mathrm{E}\left(\left|\eta_{\varepsilon, T}\right|^{p}\right)<\infty$ for all $p \in[1, \infty)$ and for all $s, t \in[0, T]$,

$$
\left|B^{H}(t, \omega)-B^{H}(s, \omega)\right| \leq \eta_{\varepsilon, T}(\omega)|t-s|^{H-\varepsilon}, \text { for any } \omega \in \Omega_{\varepsilon, T} .
$$


Transport and Communications Science Journal, Vol. 71, Issue 1 (01/2020), 11-17

We denote

$$
\tau_{n}=\inf \left\{t \in[0, T]:\left|X_{2}^{n}(t)-X_{2}^{n}(t)\right| \leq \frac{1}{n}\right\} \wedge T
$$

In order to prove that eq. (1) has a unique solution on $[0, T]$, we need the following lemma.

Lemma 2.3. The sequence $\tau_{n}$ is non-decreasing, and for almost all $\omega \in \Omega, \tau_{n}(\omega)=T$ for $n$ large enough.

Proof. Using the estimate $-(a \wedge b)=-a \vee-b$, from eq. (3) we have

$$
d\left(X_{2}^{n}(t)-X_{1}^{n}(t)\right)=\left(\frac{2 \gamma}{\left(X_{2}^{n}(t)-X_{1}^{n}(t)\right) \vee \frac{1}{n}}+b_{2}\left(t, X^{n}(t)\right)-b_{1}\left(t, X^{n}(t)\right)\right) d t+\sum_{j=1}^{m}\left(\sigma_{2 j}-\sigma_{1 j}\right) d B_{j}^{H}(t) .
$$

We set $Y^{n}(t)=X_{2}^{n}(t)-X_{1}^{n}(t)$. Eq. (4) becomes

$$
d\left(Y^{n}(t)\right)=\left(\frac{2 \gamma}{Y^{n}(t) \vee \frac{1}{n}}+b_{2}\left(t, X^{n}(t)\right)-b_{1}\left(t, X^{n}(t)\right)\right) d t+\sum_{j=1}^{m}\left(\sigma_{2 j}-\sigma_{1 j}\right) d B_{j}^{H}(t) .
$$

Then $Y^{n}(0)>0$ and $\tau_{n}=\inf \left\{t \in[0, T]:\left|Y^{n}(t)\right| \leq \frac{1}{n}\right\} \wedge T$.

It follows from Lemma 2.2 that for any $\varepsilon \in\left(0, H-\frac{1}{2}\right)$, there exist a finite random variable $\eta_{\varepsilon, T}$ and an event $\Omega_{\varepsilon, T} \in \mathcal{F}$ which do not depend on $n$ such that $\mathrm{P}\left(\Omega_{\omega, T}\right)=1$, and $\mid \sum_{j=1}^{m}\left(\sigma_{2 j}-\sigma_{1 j}\right)\left(B_{j}^{H}(t, \omega)-B_{j}^{H}(s, \omega)\left|\leq \eta_{\varepsilon, T}(\omega)\right| t-\left.s\right|^{H-\varepsilon}\right.$, for any $\omega \in \Omega_{\varepsilon, T}$ and $0 \leq s<t \leq T$.

We will adapt the contradiction method in [10]. Assume that for some $\omega_{0} \in \Omega_{\varepsilon, T}, \tau_{n}\left(\omega_{0}\right)<T$ for all $n \in \mathbb{N}$. By virtue of the continuity of sample paths of $Y^{n}$, it follows from the definition of $\tau_{n}$ that $Y^{n}\left(\tau_{n}\left(\omega_{0}\right), \omega_{0}\right)=\frac{1}{n}$ and $Y^{n}\left(t, \omega_{0}\right) \geq \frac{1}{n}$ for all $t \in\left[0, \tau_{n}\left(\omega_{0}\right)\right]$. Denote

$$
\kappa_{n}\left(\omega_{0}\right)=\sup \left\{t \in\left[0, \tau_{n}\left(\omega_{0}\right)\right]: Y^{n}\left(t, \omega_{0}\right) \geq \frac{2}{n}\right\} .
$$

We have

$$
\frac{1}{n} \leq Y^{n}\left(t, \omega_{0}\right) \leq \frac{2}{n}, \text { for all } t \in\left[\kappa_{n}\left(\omega_{0}\right), \tau_{n}\left(\omega_{0}\right)\right]
$$

In order to simplify our notations, we will omit $\omega_{0}$ in brackets in further formulas. We have $Y^{n}\left(\tau_{n}\right)-Y^{n}\left(\kappa_{n}\right)=-\frac{1}{n}=\int_{\kappa_{n}}^{\tau_{n}}\left(\frac{2 \gamma}{Y^{n}(s)}+b_{2}\left(s, X^{n}(s)\right)-b_{1}\left(s, X^{n}(s)\right)\right) d s+\sum_{j=1}^{m}\left(\sigma_{2 j}-\sigma_{1 j}\right)\left(B_{j}^{H}\left(\tau_{n}\right)-B_{j}^{H}\left(\kappa_{n}\right)\right)$.

This implies 


$$
\left|\sum_{j=1}^{m}\left(\sigma_{2 j}-\sigma_{i j}\right)\left(B_{j}^{H}\left(\tau_{n}\right)-B_{j}^{H}\left(\kappa_{n}\right)\right)\right|=\mid \frac{1}{n}+\int_{\kappa_{n}}^{\tau_{n}}\left(\frac{2 \gamma}{Y^{n}(s)}+b_{2}\left(s, X^{n}(s)\right)-b_{1}\left(s, X^{n}(s)\right) d s \mid .\right.
$$

Note that for all $s \in\left[\kappa_{n}, \tau_{n}\right]$

$$
\frac{2 \gamma}{Y^{n}(s)}+b_{2}\left(s, X^{n}(s)\right)-b_{1}\left(s, X^{n}(s) \geq 4 n \gamma\right.
$$

Then for all $n \geq n_{0}=\frac{2}{Y^{n}(0)}$, it follows from eq. (7) that

$$
\left|\sum_{j=1}^{m}\left(\sigma_{2 j}-\sigma_{i j}\right)\left(B_{j}^{H}\left(\tau_{n}\right)-B_{j}^{H}\left(\kappa_{n}\right)\right)\right| \geq \frac{1}{n}+4 n \gamma\left(\tau_{n}-\kappa_{n}\right) .
$$

This fact together with eq. (6) implies that

$$
\eta_{\varepsilon, T}\left|\tau_{n}-\kappa_{n}\right|^{H-\varepsilon} \geq \frac{1}{n}+4 n \gamma\left(\tau_{n}-\kappa_{n}\right), \text { for all } n \geq n_{0}
$$

By following similar arguments in the proof of Theorem 2 in [10], we see that the inequality (8) fails for all $n$ large enough. This contradiction completes the proof of the lemma.

We consider the process $\left\{X(t)=\left(X_{1}(t), X_{2}(t)\right)\right\}_{t \geq 0}$ which satisfies equation (1). Now, we set $Y(t)=X_{2}(t)-X_{1}(t)$, then $Y(t)$ satisfies the following equation

$$
d(Y(t))=\left(\frac{2 \gamma}{Y(t)}+b_{2}(t, X(t))-b_{1}(t, X(t))\right) d t+\sum_{j=1}^{m}\left(\sigma_{2 j}-\sigma_{1 j}\right) d B_{j}^{H}(t) .
$$

Lemma 2.4. If eq. (1) has a solution then $Y(t)=X_{2}(t)-X_{1}(t)>0$ for all $t \in[0, T]$ almost surely.

Proof. We will also use the contradiction method. Assume that for some $\omega_{0} \in \Omega$, $\inf _{t \in[0, T]} Y\left(t, \omega_{0}\right)=0$. Denote $\tau=\inf \left\{t: Y\left(t, \omega_{0}\right)=0\right\}$. For each $n \geq 1$ we denote $v_{n}=\sup \left\{t<\tau: Y\left(t, \omega_{0}\right)=\frac{1}{n}\right\}$. Since $Y$ has continuous sample paths, $0<v_{n}<\tau \leq T$ and $Y\left(t, \omega_{0}\right) \in\left(0, \frac{1}{n}\right)$ for all $t \in\left(v_{n}, \tau\right)$. We have

$$
Y(\tau)-Y\left(v_{n}\right)=-\frac{1}{n}=\int_{v_{n}}^{\tau}\left(\frac{2 \gamma}{Y(s)}+b_{2}(s, X(s))-b_{1}(s, X(s))\right) d s+\sum_{j=1}^{m}\left(\sigma_{2 j}-\sigma_{i j}\right)\left(B_{j}^{H}(\tau)-B_{j}^{H}\left(v_{n}\right)\right) .
$$

Note that for all $s \in\left[v_{n}, \tau\right]$

$$
\frac{2 \gamma}{Y(s)}+b_{2}(s, X(s))-b_{1}(s, X(s)) \geq 2 n \gamma .
$$

So we have 
Transport and Communications Science Journal, Vol. 71, Issue 1 (01/2020), 11-17

$$
\left|\sum_{j=1}^{m}\left(\sigma_{2 j}-\sigma_{i j}\right)\left(B_{j}^{H}(\tau)-B_{j}^{H}\left(v_{n}\right)\right)\right| \geq \frac{1}{n}+2 n \gamma\left(\tau-v_{n}\right) .
$$

Again using the inequality (6), we have

$$
\eta_{\varepsilon, T}\left|\tau-v_{n}\right|^{H-\varepsilon} \geq \frac{1}{n}+2 n \gamma\left(\tau-v_{n}\right)
$$

Similar to the argument of Theorem 2 in [10] we see that the inequality (11) fails for all $n$ large enough. This contradiction completes the lemma. follows

Based on above lemmas we obtain the main theorem of this paper which is stated as

Theorem 2.5. For each $T>0$ eq. (1) has a unique solution on $[0, T]$.

Proof. First, from Lemma 2.3, there exists a finite random variable $n_{0}$ such that $X_{2}^{n}(t)-X_{2}^{n}(t) \geq \frac{1}{n_{0}}>0 \quad$ almost surely for any $t \in[0, T]$. Therefore, the process $X^{n}(t)=\left(X_{2}^{n}(t), X_{2}^{n}(t)\right)$ converges almost surely to a limit, called $X(t)$ when $n$ tends to infinity and $X(t)$ satisfies eq. (1). This fact together with Lemma (2.4) leads to eq. (1) has a strong non- colliding solution.

Next, we show that eq. (1) has a unique solution in path-wise sense. Let $X(t)$ and $\bar{X}(t)$ be two solutions of eq. (1) on $[0, T]$. We have

$$
\begin{aligned}
& \left|X_{1}(t, \omega)-\overline{X_{1}}(t, \omega)\right|= \\
& =\left|\int_{0}^{t}\left(\frac{\gamma}{X_{1}(s, \omega)-X_{2}(s, \omega)}+b_{1}(s, X(s, \omega))-\frac{\gamma}{\overline{X_{1}}(s, \omega)-\overline{X_{2}}(s, \omega)}-b_{1}(s, \bar{X}(s, \omega))\right) d s\right| \\
& \leq \int_{0}^{t}\left(\frac{\gamma}{X_{1}(s, \omega)-X_{2}(s, \omega)}-\frac{\gamma}{\overline{X_{1}}(s, \omega)-\overline{X_{2}}(s, \omega)}\right)\left|d s+\int_{0}^{t}\right| b_{1}(s, X(s, \omega))-b_{1}(s, \bar{X}(s, \omega)) \mid d s .
\end{aligned}
$$

Using the continuous property of the sample paths of $X(t)$ and $\bar{X}(t)$ and Lemma 2.4, we have

$$
m_{0}=\min _{t \in[0, T]}\left\{X_{2}(t, \omega)-X_{1}(t, \omega), \bar{X}_{2}(t, \omega)-\bar{X}_{1}(t, \omega)\right\}>0 .
$$

This fact together with the Lipschitz condition of $b$ leads to

$$
\left|X_{1}(t, \omega)-\overline{X_{1}}(t, \omega)\right| \leq \int_{0}^{t} \frac{\gamma\left|\left(X_{2}(s, \omega)-\bar{X}_{2}(s, \omega)\right)-\left(X_{1}(s, \omega)-\bar{X}_{1}(s, \omega)\right)\right|}{m_{0}^{2}}+\int_{0}^{t} L|X(s, \omega)-\bar{X}(s, \omega)| d s .
$$

Similarly, we estimate $\left|X_{2}(t, \omega)-\overline{X_{2}}(t, \omega)\right|$. We obtain 


$$
\sum_{i=1}^{2}\left|X_{i}(t, \omega)-\bar{X}_{i}(t, \omega)\right| \leq\left(\frac{2 \gamma}{m_{0}^{2}}+2 L\right) \int_{0}^{t} \sum_{i=1}^{2}\left|X_{i}(s, \omega)-\overline{X_{i}}(s, \omega)\right| d s .
$$

It follows from Gronwall's inequality that

$$
\sum_{i=1}^{2}\left|X_{i}(t, \omega)-\overline{X_{i}}(t, \omega)\right|=0, \text { for all } t \in[0, T] \text {. }
$$

Therefore, $X(t, \omega)=\bar{X}(t, \omega)$ for all $t \in[0, T]$. The uniqueness has been concluded.

\section{CONCLUSION}

The main result proved in this paper is the existence and uniqueness of strong noncolliding solution in path- wise sense to the two- dimensional fractional non- colliding particle systems. From this result, we can propose a numerical approximation for this system.

\section{REFERENCES}

[1] P. Kloeden, E. Platen, Numerical solution of stochastic differential equations, SpringerVerlag, 1995.

[2] M. Katori, H. Tanemura, Noncolliding Squared Bessel processes, J. Stat. Phys., 142 (2011) 592615. https://doi.org/10.1007/s10955-011-0117-y

[3] M. Katori, H. Tanemura, Noncolliding processes, matrix-valued processes and determinantal processes, Sugaku Expositions, 24 (2011) 263-289. https://doi.org/10.11429/sugaku.0613225

[4] E. Cepa, D. Lepingle, Diffusing particles with electrostatic repulsion, Probab.Theory Related Fields, 107 (1997) 429-449. https://doi.org/10.1007/s004400050092

[5] P. Graczyk, J. Ma lecki, Strong solutions of non-colliding particle systems, Electron. J. Probab, 19(2014) 1-21.

[6] L. C. G. Rogers, Z. Shi, Interacting Brownian particles and the Wigner law, Probab. Theory Related Fields, 95 (1993) 555-570. https://doi.org/10.1007/BF01196734

[7] N. Naganuma, D. Taguchi, Malliavin Calculus for Non-colliding Particle Systems, Stochastic Processes and their Applications, 2019. https://doi.org/10.1016/j.spa.2019.07.005

[8] D. Nualart, A. Rascanu, Differential equations driven by fractional Brownian motion, Collectanea Mathematica, 53 (2002) 177-193.

[9] Y. S. Mishura, Stochastic Calculus for Fractional Brownian Motion and Related Processes, Lecture Notes in Math, Springer, Berlin, 2008.

[10] Y.. Mishura, A. Yurchenko-Tytarenko, Fractional Cox-Ingersoll-Ross process with non-zero "mean", Modern Stochastic: Theory and Applications, 5 (2018) 99-111. https://doi.org/10.15559/18VMSTA97 